\section{Ethical Analysis of Communicating the Risk of Iatrogenic Creutzfeldt-Jakob Disease}

To the Editor-The University of Michigan Health System Adult Ethics Committee recently convened to discuss whether 57 patients should be informed of possible exposure to classic Creutzfeldt-Jakob disease (CJD) after a neurosurgical patient was suspected of contracting CJD. Current routine sterilization procedures are believed to be ineffective against prions; thus, subsequent neurosurgical patients were potentially at risk of contracting CJD from contaminated instruments. AIthough eventually the patient definitively tested negative for $\mathrm{CJD}$, the committee's discussion raised several key issues regarding communication of the risk of iatrogenic transmission of prion disease.

The majority of known cases of iatrogenic transmission of sporadic CID have been from the use of human growth hormone or from dural grafts, with over 100 known cases by each route of transmission. The posttransplant incubation period before CJD develops ranges from 2 to 30 years. ${ }^{1,2}$ Iatrogenic transmission of CJD from surgical instruments has been documented in only 6 cases since 1950 . The evidence for many of these cases of transmission was largely circumstantial: an iatrogenic origin was presumed when a patient was readmitted to the hospital with neurological deterioration 18-24 months after surgery. The average time from presumed exposure to symptomatic disease was 18 months; however, cases with longer incubation periods may remain unrecognized. Although the Centers for Disease Control and Prevention estimate that fewer than $1 \%$ of cases of sporadic CJD can be attributed to contaminated surgical instruments, ${ }^{3}$ a recent population-based study suggests that the percentage may be as high as $18 \%{ }^{4}$ Of note, the literature suggests that the development of symptoms may be significantly more rapid after iatrogenic transmission than would be expected in sporadic cases.

Conventional sterilization procedures for surgical equip-

TABLE. Health-Related Quality of Life Assessment for Patients in the 2 Treatment Groups, April 2006 to March 2007

\begin{tabular}{lccc}
\hline & \multicolumn{2}{c}{ Mean value \pm standard deviation } & \\
\cline { 2 - 4 } Variable & $\begin{array}{c}\text { Ciprofloxacin group } \\
(n=24)\end{array}$ & $\begin{array}{c}\text { Tazocin group } \\
(n=23)\end{array}$ & $P^{\mathrm{a}}$ \\
\hline EQ-5D score & $0.50 \pm 0.35$ & $0.54 \pm 0.31$ & .669 \\
EQ-VAS score & $56.00 \pm 16.67$ & $65.40 \pm 18.67$ & .075 \\
\hline
\end{tabular}

NOTE. EQ-5D, EuroQol Group 5-dimensional questionnaire; EQ-VAS, EuroQol Group visual analogue scale.

a $P \leqslant .05$ was considered significant. ment do not eradicate prions, although repeated sterilization cycles lessen infectivity. ${ }^{5}$ Recommendations for complete disinfection with sodium hydroxide or bleach have been endorsed by the World Health Organization ${ }^{6}$; however, some instruments are too delicate for use with these harsh agents and too expensive to discard after each surgery. Potent disinfectants also carry some risk to staff. Consequently, these protocols are not in widespread use. Furthermore, in many cases, CJD may not be suspected until after the patient undergoes surgery, making special perioperative measures impossible. Under the current standard of care, therefore, any neurosurgical patient is theoretically at risk for CJD.

Knowledge of exposure to CJD is currently of very little clinical use, because no definitive tests are available to determine whether exposed patients are infected, no pharmacologic agents are available to halt the development of CJD, and no treatments are available once it is determined that clinical disease is present. In this way, exposure to prion disease differs from exposure to infectious agents such as the human immunodeficiency virus. However, clinical use may not be the most appropriate criterion to determine whether patients should be informed of exposure. Patients may also use risk information to help them determine how to manage other important aspects of their lives, such as updating advance directives and making educational, legal, or career plans based on the possibility of developing clinical disease. Although risk of transmission of CJD among family members is considered negligible, ${ }^{3}$ patients notified of exposure could presumptively request surgical precautions to limit the risk of exposing other patients to CJD.

Some committee members were concerned that the psychological distress caused by awareness of the risk of developing a fatal, untreatable disease would outweigh the potential benefits of receiving such information. However, it is important to note that, whether or not the distress caused by notification of exposure to CJD is in fact significant, information is not usually withheld in the healthcare setting simply because it may be upsetting. Additionally, patients often adjust to adverse circumstances better than providers may expect; patients with chronic disease show remarkable adaptability to decreases in functional status, ${ }^{7}$ and individuals receiving unfavorable results of genetic testing show strong coping skills and tend to exhibit less emotional distress than anticipated by clinicians. ${ }^{8}$

It is standard practice to communicate desired information about medical care to patients, and it is equally established that, in most situations, undesired information should not be communicated. Ideally, then, patients' preferences to receive risk information would be determined prior to deciding on a disclosure policy. Unfortunately, this approach may not be feasible on a case-by-case basis.

The committee's best estimate suggested that, on the basis 
of the paucity of documented cases of transmission of CJD during surgery, the quantitative risk of transmission of CJD was exceedingly low; however, the study by Mahillo-Fernandez et $\mathrm{al}^{4}$ suggesting that 1 in 5 CJD cases may be attributable to surgical intervention had not yet been published. The risk of iatrogenic transmission of CJD should be considered both in the context of spontaneous incidence of CJD (approximately 1 in 1,000,000 persons) and in the context of the significantly greater risk of other serious nosocomial events (eg, the development of antibiotic-resistant infections) not routinely highlighted on hospital admission. It may accordingly be argued that the risk of iatrogenic transmission of CJD is covered in the standard consent form signed by patients upon hospitalization. However, patients may consider the risk of exposure to CJD to be qualitatively different from other hospital-related risks, because of the rarity of the disease and the prominence given to variant CJD in the media. This is an empirical question that has not been adequately addressed.

Finally, the committee considered the effect that disclosure may have on the reputation of our health system, which has always had a goal of transparency. Public perception of a failure to adequately protect patients from iatrogenic infection may constitute negative publicity; however, failure to disclose may be perceived as even more egregious. Nondisclosure could be defended in the context of other hospital policies regarding risk communication; however, transparency could increase patients' trust in the healthcare system over the long term and strengthen the doctor-patient relationship.

Although the negative test results of the index patient rendered this case moot, the University of Michigan Health System implemented an instrument-tracking system for neurosurgical instruments. In the future, fewer patients will be at risk if a potentially contaminated instrument can be identified and isolated.

This case highlights the need for research in several areas. First, surprisingly little is known about the qualitative and quantitative thresholds above which patients want to be informed of risks associated with their medical care. Patients' desires for information on exposure risk should be assessed, including the risk of contracting an infectious disease with limited diagnostic and treatment options. Second, future research should address the impact on patients of communicating the risk of exposure to disease, including the positive and negative psychological consequences, the potential impact of disclosure on future health behaviors, and the methods of understandably communicating the risk of disease transmission. Third, the implications of disclosure policies on the public perception of healthcare institutions should be investigated. This research should foster an open and honest discussion among institutions of possible approaches to communicating unforeseen exposures. Fourth, an accurate assessment of the risk of iatrogenic transmission of prion disease among neurosurgical patients is essential for the development of infection control guidelines and the education of the public. Finally, all of the above should be integrated into research that critically examines a hospital's responsibility to disclose the risk of transmission of disease, including which risks can reasonably be integrated into boilerplate consent documents for hospital admission or surgical procedures. Although it may be impossible to provide risk-free health care for patients, ${ }^{9}$ minimizing to the greatest feasible extent the risk of contracting an infectious disease, in conjunction with providing transparency about current risks, will likely contribute to increased patient satisfaction and improved health outcomes.

\section{ACKNOWLEDGMENTS}

We thank Sandro Cinti and Paul McKeever for comments that were helpful in the development of this manuscript.

Potential conflicts of interest. All authors report no conflicts of interest relevant to this article.

David I. Shalowitz, AB; Andrew Barnosky, DO, MPH; Lauren Barrett Smith, MD

From the Bioethics Program (D.I.S.), the Department of Emergency Medicine (A.B.), and the Department of Pathology (L.B.S.), University of Michigan Medical School, Ann Arbor, Michigan.

Address reprint request to David I. Shalowitz, AB, Bioethics Program, University of Michigan, Ann Arbor, MI 48109 (dshalowi@med.umich.edu). Infect Control Hosp Epidemiol 2009; 30:805-806

(C) 2009 by The Society for Healthcare Epidemiology of America. All rights reserved. 0899-823X/2009/3009-0018\$15.00. DOI: 10.1086/599004

\section{REFERENCES}

1. Sutton JM, Dickinson J, Walker JT and Raven ND. Methods to minimize the risks of Creutzfeldt-Jakob disease transmission by surgical procedures: where to set the standard? Clin Infect Dis 2006; 43:757-764.

2. Update: Creutzfeldt-Jakob disease associated with cadaveric dura mater grafts-Japan, 1978-2008. MMWR Morb Mortal Wkly Rep 2008; 57:11521154.

3. Centers for Disease Control and Prevention (CDC). CJD (CreutzfeldtJakob disease, classic). Information on Creutzfeldt-Jakob disease for funeral home, cemetery, and crematory practitioners. Available at: http:// www.cdc.gov/ncidod/dvrd/cjd/funeral_directors.htm. Accessed June 15, 2009.

4. Mahillo-Fernandez I, de Pedro-Cuesta J, Bleda MJ, et al. Surgery and risk of sporadic Creutzfeldt-Jakob disease in Denmark and Sweden: registrybased case-control studies. Neuroepidemiology 2008; 31:229-240.

5. Fichet G, Comoy E, Duval C, et al. Novel methods for disinfection of prion-contaminated medical devices. Lancet 2004; 364:521-526.

6. Taylor DM. Inactivation of prions by physical and chemical means. J Hosp Infect 1999; 43 Suppl:S69-S76.

7. Damschroder LJ, Zikmund-Fisher BJ, Ubel PA. Considering adaptation in preference elicitations. Health Psychol 2008; 27:394-399.

8. Broadstock M, Michie S, Marteau T. Psychological consequences of predictive genetic testing: a systematic review. Eur J Hum Genet 2000; 8:731738.

9. Amalberti R, Auroy Y, Berwick D, Barach P. Five system barriers to achieving ultrasafe health care. Ann Intern Med 2005; 142:756-764. 